\title{
Benign Lymphoepithelial Cyst in An Adolescent Female Mimicking Lymphoma: A Diagnostic Dilemma in a Retrovirus Negative Patient
}

\author{
Somshankar Chowdhury, Sufian Zaheer*, Preeti Sharma and Ashish Kumar Mandal
}

Department of Pathology, Vardhman Mahavir Medical College and Safdarjung Hospital, New Delhi- India

\begin{abstract}
Cystic lesions of the parotid gland are uncommon and may masqueradeneoplasms clinically. Benign lympho-epithelial cysts (BLEC) comprise the most common causes of the parotid gland swelling in human immunodeficiency virus (HIV)-positive patients. It is, however, less common amongst those not infected with HIV. We present a case of a12 years old female who presented with a swelling over left parotid region since one year.Local examination revealed a soft, cystic and non-tender swelling in the left parotid region measuring $5 \mathrm{x} 4 \mathrm{x} 3$ $\mathrm{cm}$. Serological investigations for viral markers were non-reactive.A differential diagnosis of granulomatous parotitis versus benign lymphoepithelial lesion was rendered on radiological investigations and superficial parotidectomy was planned. On histopathological examination, a cyst lined by cuboidal to low columnar lining was seen, underlying which, was dense lympho-plasmacytic infiltrate present in diffuse sheets. On immunohistochemistry, this lymphoid population was found to be reactive in nature (positive for kappa and lambda), thus excluding lymphoma. This case is being presented due to its rarity in HIV negative individuals and the histopathological diagnostic dilemma it presented with, mimicking a lymphoma.
\end{abstract}

Keywords: Lymphoepithelial Cyst; Parotid; Lymphoma; HIV

\section{Introduction}

Cystic lesions within the parotid gland are uncommon, comprising approximately $5 \%$ of all salivary gland tumours, many of them representingcystic components of neoplasms. ${ }^{[1]}$ Lymphoepithelial (LE) cysts are known to arise in the lateral cervical areas, referred to as branchial cleft cysts, or in the oral mucosa. ${ }^{[2]}$ Albeit rare, the parotid gland has been recognized as a site for these unusual lesions. ${ }^{[3]}$

The term "lymphoepithelial cyst" was denominated by Bernier and Bhaskar to lay emphasis on the non-embryonic derivation of this lesion. ${ }^{[4]}$ These cysts are usually seen inhuman immunodeficiency virus (HIV) positive individuals and their rise in incidence has coincided with that of HIV infection, occasionally as the first manifestation of retroviral infection. ${ }^{[5,6]}$ However, they have also been seen in HIV negative individuals, although less frequently, thereby making its pathogenesis elusive with respect to HIV. ${ }^{[6,7]}$

Lesions of salivary glands with a prominent lymphoid component are a heterogeneous group of diseases that include benign as well as malignant lesions. These entities may, at times, pose difficulties in diagnosis. Lymphoepithelial sialadenitis, Warthin's tumor, extranodal marginal zone B-cell lymphoma, chronic sclerosing sialadenitis and HIVassociated salivary gland disease including lymphoepithelial cysts are few such entities that might confound the clinician as well as the pathologist. Our case highlights the diagnostic dilemma associated with lymphoepithelial cysts especially in retrovirus negative patients.

\section{Case Report}

A 12 years old female child presented in the out-patient department with complaints of a swelling over left parotid region. The swelling appeared one year ago and gradually increased in size to attain its present dimension of $5 \times 4 \times 3 \mathrm{~cm}$. The overlying skin appeared unremarkable. There was no history of any pain while taking food. On examination, the swelling was soft, cystic and non-tender. No signs of facial nerve involvement was present. General physical examination and systemic examination was within normal limits. All the routine blood investigations including the haemogram, liver function tests and kidney function tests were within normal limits. Serological investigations for viral markers were nonreactive. Ultrasonography revealed bilateral bulky parotid glands (left >> right) with multiple intraparotid cystic lesions, the largest of which was $3.8 \times 3.2 \mathrm{~cm}$, within the left parotid. A differential diagnosis of granulomatous parotitis versus benign lymphoepithelial lesion was given. Magnetic resonance imaging (MRI) scan suggested the possibility of an impacted branchial cyst (Figure 1a, b).

Fine needle aspiration yielded thin fluid. Smears were paucicellular with few inflammatory cells and macrophages against a fluid background. With a possibility 
of a lymphoepithelial cyst, superficial parotidectomy was performed and sent for histopathological examination. The immediate post-operative period was uneventful.

Gross examination revealed a grey brown encapsulated cystic structure, measuring $4.5 \times 3.5 \times 2 \mathrm{~cm}$. On cut, a cyst was identified, filled with mucinous fluid. The wall thickness varied from 0.1 to $0.2 \mathrm{~cm}$. Microscopic examination revealed a cyst lined by cuboidal to low columnar cells and surrounded by fibrocollagenous tissue. Beneath the lining were diffuse sheets of dense lymphoid infiltrate intermixed with plasma cells and macrophages (Figure 2a-d). At places, germinal centre formation was seen. No lymphoepithelial lesions were seen. Owing to the dense and diffuse lymphoid infiltrate, an immunohistochemical panel was applied to rule out the possibility of a lymphoma. On immunohistochemistry, the lymphoid cells were diffusely positive for leucocyte common antigen (LCA). Immunoreactivity for $\mathrm{CD} 20$ was observed within the germinal centres (Figure 3a) and for CD3 in the para-follicular areas (Figure 3b). Polyclonal expression of Kappa (Figure 3c) and lambda (Figure 3d) expression excluded the possibility of lymphoma.Based on the radiological, histo-morphological and immunohistochemical findings a final diagnosis of benign lympho-epithelial cyst of left parotid gland was rendered.On follow up, patient is currently healthy one year after excision.

\section{Discussion}

Branchial cyst in the parotid gland was first reported in 1895 by Hildebrant. BLEC otherwise quite rare in general, is found most commonly in HIV-positive patients. Thus, a patient diagnosed with these cysts, should be tested for HIV, if the status is previously unknown. These cysts, when in HIV positive individuals, are bilateral in $80 \%$ cases and multiloculated in $90 \%$ cases. ${ }^{[8,9]}$ In our case, the cyst was unilateral and uniloculated, both features being in consistency with the HIV negative status of the patient.

The age of patients in various case reports have ranged from 16 to 69 with the mean age of 44 years old. ${ }^{[10]}$ Our patient was slightly younger than any of the reported cases, being 12 years of age.

Histopathological examination is the gold standard for diagnosis of lymphoepithelial cysts. The dense lymphocytic component of these cysts often creates a diagnostic dilemma and a variety of differential diagnoses need to considered. Lymphoepithelial sialadenitis, Warthin's tumour, extranodal marginal zone B-cell lymphoma and chronic sclerosing sialadenitis are some of the lesions that need to be distinguished from lymphoepithelial cysts.

The most common lymphoma arising from salivary glands is extra-nodal marginal zone B cell lymphoma. Follicular and diffuse large B-cell lymphomas are the other common

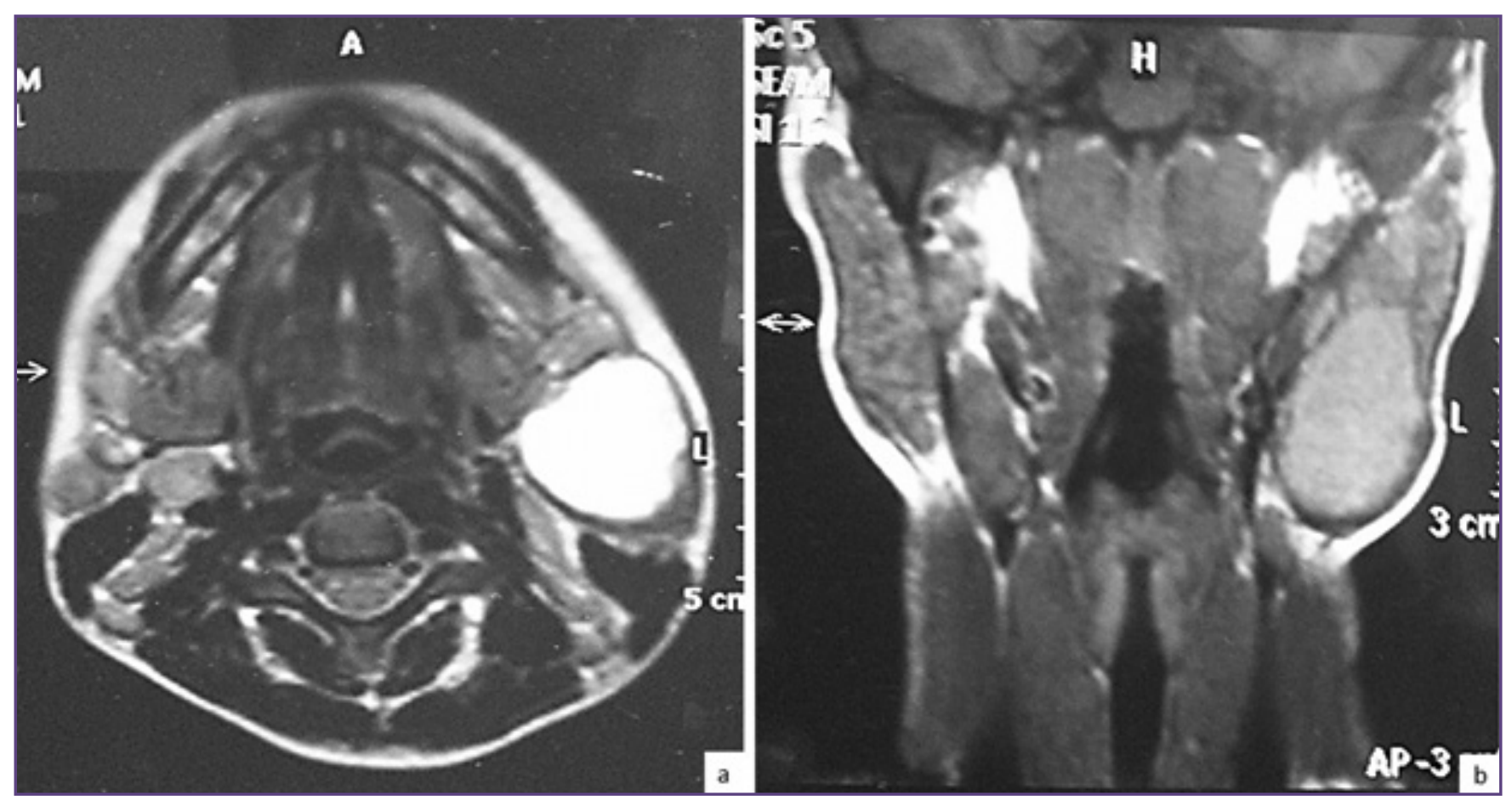

Fig. 1: (a\& b)MRI neck showed evidence of an altered signal intensity lesion measuring $3.2 \times 2.7 \mathrm{~cm}$ in the left parotid suggestive of infected branchial cyst. 


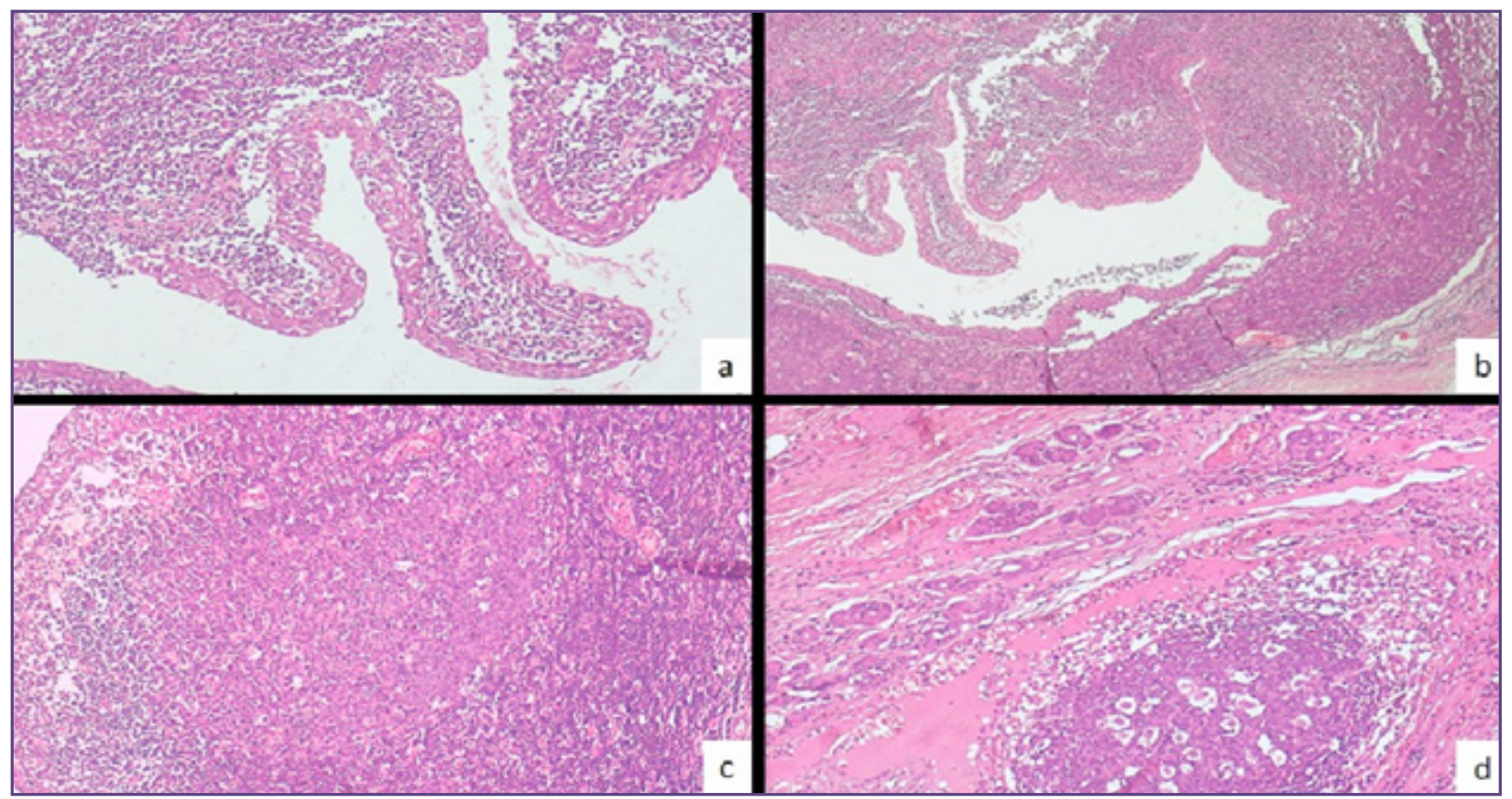

Fig. 2: (a\& b) Photomicrograph showing cyst wall lined by cuboidal to low columnar cells underneath which is dense lymphoid infiltrate (H\&E; x100) (c) Lymphoid infiltrate seen in diffuse sheets (H\&E; x200) (d) Surrounding parotid gland acini showing presence of similar lymphoid infiltrate (H\&E; x200).
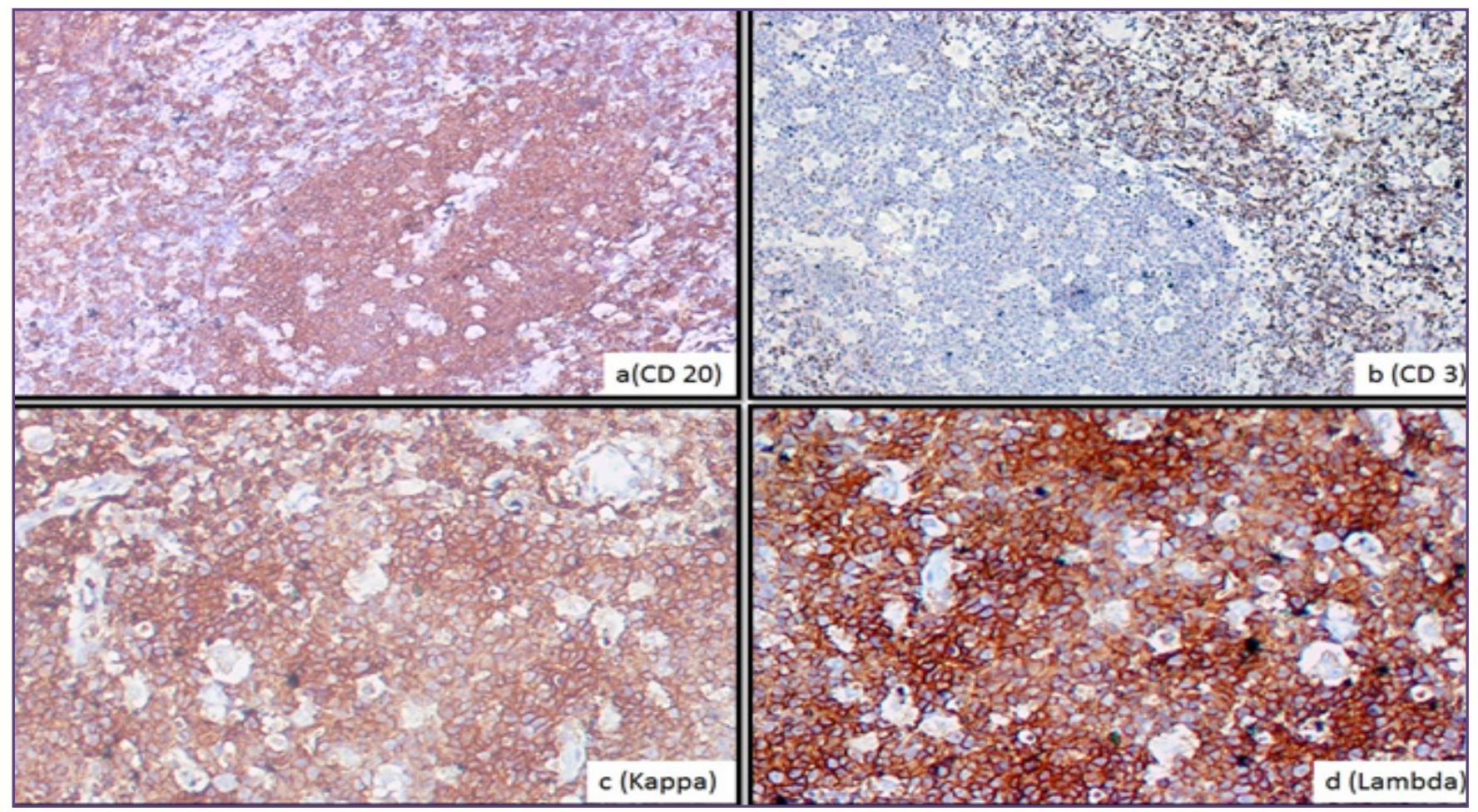

Fig. 3: (a\& b) Photomicrograph showing immunoreactivity for (a) CD 20 in the germinal centres (CD 20 immunostain x200)

(b) CD 3 in the para-follicular regions (CD 3 immunostain x100) (c) Kappa in all lymphoid cells(Kappa immunostain x400) (d) Lambda in all lymphoid cells (Lambda immunostain $x 400$ ). 
ones. ${ }^{[11]}$ Most of the marginal zone B-cell lymphomas (MZBCL) arise against a background pre-existing lymphoepithelial sialadenitis. A characteristic of diagnostic utility in such emerging MZBCLs is the presence of lymphoepithelial lesions. These are hyperplastic ductal epithelium, with the epithelial cells permeated by neoplastic lymphocytes. Surrounding these lesions, are proliferations of neoplastic marginal zone lymphoma cells. Admixed with the neoplastic lymphocytic proliferation is a nonneoplastic infiltrate comprising T-cells, B-cells, plasma cells, reactive germinal centers, and scattered centroblasts or immunoblasts. With such a picture, lymphoepithelial sialadenitis and lymphoepithelial cysts are the two principal considerations in the differential diagnosis. Our panel of immunohistochemistry showed polyclonal nature of the lymphoid cells, and a mixture of B cells in the germinal centres and $\mathrm{T}$ cells in the interfollicular areas.

Lymphoepithelial sialadenitis is characterized by a benign lymphocytic infiltrate of salivary gland with parenchymal atrophy, ductal hyperplasia and lymphocytic epitheliotropism. Lymphoepithelial lesions are often prominent. It usually presents as a component of the autoimmune condition of Sjogren syndrome. Although T cells comprise the predominant population of lymphocytes, the lymphocytes infiltrating into the ductal epithelial cells are of B cell type. These patients have a much higher risk of developing lymphoma, and most, if not all, salivary extranodal MZBCLs are preceded by lymphoepithelial sialadenitis. Distinction from MZBCL is based on the number and distribution of marginal zone B cells.

The origin of these cysts is controversial and four theories have been suggested till date. ${ }^{[12]}$ The branchial apparatus theory and the cervical sinus theory, also known as classic theory, suggest that the cysts develop from the remnants of the branchial cleft. The Inclusion Theory, however, believes that the cysts arise from cystic changes in parotid gland epithelium that become entrapped in the upper cervical lymph nodes during embryonic life. ${ }^{[4]}$ The pathogenesis of these cysts in HIV positive individuals is related to the "Ductal obstruction phenomena" where there is obstruction of parotid ducts along with a follicular hyperplasia in the periductal parotid lymph nodes, induced by the virus.

To conclude, lymphoepithelial cysts are uncommonly encountered lesions of the parotid gland which are rarer in children and retroviral negative patients. Furthermore, these cysts masquerade neoplasms of the salivary gland clinicoradiologically with histopathology being the gold standard for a decisive diagnosis. Our caseemphasises that this lesion should be kept in mind as a differential diagnosis while dealing with lymphoproliferative lesions of the parotid gland.

\section{References}

1. Altman K and Bailey MW. Parotid cyst: a case report. Int J Oral Maxillofac Surg 1994;23:165-166.

2. Neville BW, Damm DD, Allen CM, Bouquot JE. Oral and maxillofacial pathology. 3rd ed. St Louis: Mosby;2008.

3. Terry JH, Loree TR, Thomas MD, Marti JR. Major salivary gland lymphoepithelial lesions and the acquired immunodeficiency syndrome. Am J Surg 1991;162:324-329.

4. Bernier JL, Bhaskar SN. Lymphoepithelial lesions of salivary glands. Histogenesis and classification based on 186 cases. Cancer 1958;11:1156-1179.

5. Ihrler S, Zietz C, Riederer A, Diebold J, Löhrs U. HIV-related parotid lymphoepithelial cysts. Immunohistochemistry and 3 -D reconstruction of surgical and autopsy material with special reference to formal pathogenesis. Virchows Arch 1996;429:139-147.

6. Maiorano E, Favia G, Viale G. Lymphoepithelial cysts of salivary glands: an immunohistochemical study of HIV-related and HIV unrelated lesions. HUM PATHOL 1998;29:260-265.

7. Chetty R. HIV-associated lymphoepithelial cysts and lesions:morphological and immunohistochemical study of the lymphoid cells. Histopathology 1998;33:222-229.

8. Dave SP, Pernas FG, Roy S. The benign lymphoepithelial cyst and a classification system for lymphocytic parotid gland enlargement in the pediatric HIV population. Laryngoscope 2007;117(1):106-113.

9. Kooper DP, Leemans CR, Hulshof MC,et al. Management of benign lymphoepithelial lesions of the parotid gland in human immunodeficiency virus-positive patients. Eur Arch Otorhinolaryngol 1998;255(8):427-429.

10. Rahman S, Shaari R, Hassan R. Parotid lymphoepithelial cyst: A case report. Archives of Orofacial Sciences 2006;1:71-75

11. Ahamed AS, Kannan VS, Velaven K,et al. Lymphoepithelial cyst of the submandibular gland. J Pharm Bioallied Sci 2014;6:185-187.

12. Camilleri AC, Lloyd RE. Lymphoepithelial cyst of the parotid gland. Br J Oral Maxillofac Surg 1990;28:329-332.

*Corresponding author:

Sufian Zaheer, MD Pathology, Associate Professor, Department of Pathology, Vardhman Mahavir Medical College and Safdarjung Hospital, New Delhi, India

Phone: +91 9650594818

Email: sufianzaheer@gmail.com

Financial or other Competing Interests: None.

Date of Submission : 01.11.2016

Date of Acceptance : 20.04.2017

Date of Publication : 25.08.2017 